\title{
Three-Pass Protocol Concept in Hill Cipher Encryption Technique
}

\author{
Andysah Putera Utama Siahaan \\ Faculty of Computer Science, Universitas Pembangunan Panca Budi \\ J1. Jend. Gatot Subroto Km. 4,5 Sei Sikambing, 20122, Medan, Sumatera Utara, Indonesia
}

\begin{abstract}
Hill Cipher Encryption technique has a square matrix in its calculation. The ciphertext resulted is obtained from the matrix multiplication between plaintext and key. The reality is the sender must send or tell the receiver the key used to encrypt the data before the receiver can decode the ciphertext into the legible message. The listener is able to read the key that flows to the receiver. By knowing the key, the listener will absolutely break the ciphertext and turn into plaintext. Three-Pass Protocol is a way to limit the key being distributed. The sender and receiver have their own keys in hand. They do not need to share each other. This method will improve the security.
\end{abstract}

Keywords: Cryptography, Three-Pass Protocol, Hill Cipher, Encryption, Decryption

\section{Introduction}

The confidential information must be protected from being intercepted [9]. There is a various way to perform the encryption. Hill Cipher is one of the encryption algorithms that uses matrix [8][7]. The smallest matrix of $2 \times 2$ can produce the ciphertext by providing key as the determinant. The matrix bigger than $2 \times 2$ can be used as well, but the difficulties in finding the inverse matrix gain more too. In Hill Cipher, we can randomly describe the integers for keys beforehand [5][6]. However, sometimes the key provided does not work. It happens when decoding the ciphertext back to plaintext. It is different from the original message. Before using the key, we have to test that it has the right determinant. Moreover, if so, the inverse key will be applied to the ciphertext when decryption is happening. Since we use the key as a password to modify the message, we have to send or give to someone who is responsible for decrypting the message. The key must be distributed, and this moment will be taken by third parties to intercept the known plaintext to be breakable. Three-Pass Protocol is the best way to reduce the gap of interception. On the application of this algorithm, the form of the matrix must be modified. The are several changes of Hill Cipher part to make the both algorithms work together.

\section{Theories}

The symmetric key is one of the cryptographic systems that uses the same kind of keys in encryption and decryption. Hill Cipher uses the symmetric key in its application. However, the keys used in encryption and decryption are different but same. It happens because the key used in decryption is the inverse of the original key applied when sending plaintext to the receiver [2][3]. The both keys must be correctly calculated for them to generate encrypt and decrypt key pair in encryption and decryption works.

Hill Cipher is an application of modulo arithmetic in cryptography [4]. This cryptographic technique uses the matrix as the vessel of information exchange either on encryption or decryption part. The basic theory of matrix used in Hill Cipher is the multiplication between the matrix and the inverse of the matrix. Hill Cipher is a symmetric key hard to solve because the cryptanalysis techniques such as frequency analysis can not be applied easily to solve this algorithm [2]. Hill cipher is very difficult to solve if cryptologist has only the ciphertext, but it can be solved easily if the cryptologist has a part of the plaintext.

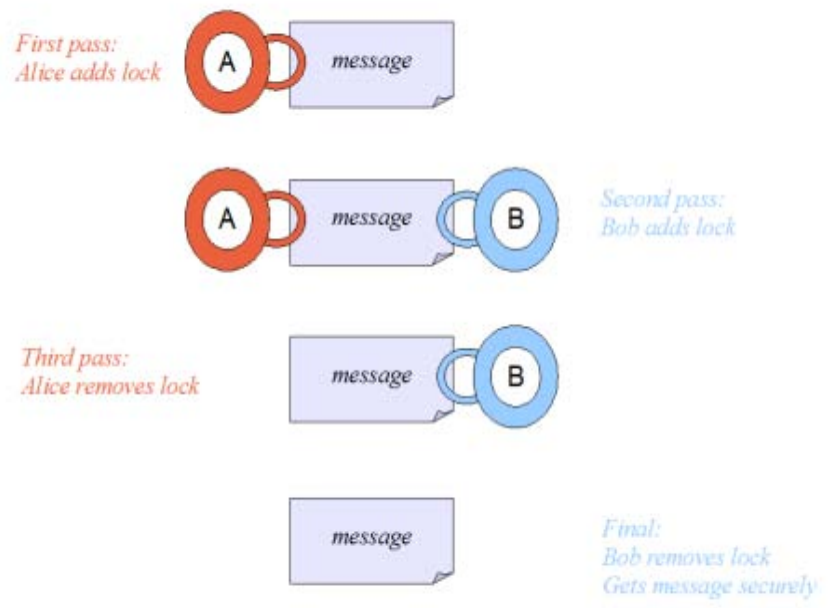

Figure 1:The Three-Pass Protocol scheme

Three-Pass Protocol method is a way to send a message securely from sender to receiver without the need to exchange or distribute encryption keys [1]. In Three-Pass Protocol, the sender encrypts the message using a unique encryption key then they send it to the receiving participant. When the receiver gets the encrypted message, they then encrypt it with their own unique encryption key and send back to the sender. Then the sender decrypts the message with their own key. After this, there is only one level of encryption on the package which is sent to the receiver who decrypts the final layer with their unique decryption key and reads the data. This protocol can only be used if using commutative ciphers or LIFO method. Commutative means that the order of encryption and decryption is interchangeable (Encryption A - Encryption B - Decryption A - Decryption B) [4](Figure 1). 


\section{International Journal of Science and Research (IJSR) \\ ISSN (Online): 2319-7064}

Index Copernicus Value (2013): 6.14 | Impact Factor (2015): 6.391

\section{Proposed Work}

In the application of Three-Pass Protocol in Hill Cipher, the plaintext cannot directly transform to ciphertext and then reencrypt the message with the second key. If we do this when doing the decryption, the message will not turn back to its original message, it turns to different characters order. We have to modify the encryption block with a square block. It means, when we use a key of a matrix of $2 \times 2$, the plaintext block will be $2 \times 2$ as well. It is totally different from the usual Hill Cipher encryption that uses different matrix order. $C=\left(\begin{array}{ll}a & b \\ c & d\end{array}\right) \times\left(\begin{array}{ll}p 1 & p 3 \\ p 2 & p 4\end{array}\right)$ mod TotalCharacter $D=\left(\begin{array}{ll}a & b \\ c & d\end{array}\right)^{-1} x\left(\begin{array}{ll}c 1 & c 3 \\ c 2 & c 4\end{array}\right)$ mod TotalCharacter

From the formulas above, the encryption and decryption are using the same blocks with the key.

\section{Testing and Implementation}

In this section, we try to prove the Three-Pass Protocol. Let's take an example below:

$\begin{array}{lll}\text { Plaintext } & : & \operatorname{ANDY}\left(\begin{array}{cc}65 & 68 \\ 78 & 89\end{array}\right) \\ \text { Key 1 } & : & \left(\begin{array}{cc}240 & 97 \\ 65 & 163\end{array}\right) \\ \text { Key 2 } & : & \left(\begin{array}{cc}187 & 23 \\ 148 & 223\end{array}\right) \\ {\text { Key } 1^{-1}}^{2} & : & \left(\begin{array}{cc}205 & 145 \\ 113 & 16\end{array}\right) \\ \text { Key 2 }^{-1} & : & \left(\begin{array}{cc}55 & 209 \\ 76 & 115\end{array}\right)\end{array}$

Now we prove that the keys provided are invertible.

$\begin{array}{lll}\text { Key } 1 \quad: & \left(\begin{array}{cc}240 & 97 \\ 65 & 163\end{array}\right) \\ & & \\ \text { Determinant }: & (240 * 163-97 * 65) \text { mod } 256 \\ & 47(\mathrm{D} \neq 0 \text { and } \mathrm{D} \neq \text { Even }) \\ \text { Key } 2 \quad: & \left(\begin{array}{cc}187 & 23 \\ 148 & 223\end{array}\right) \\ \text { Determinant }: & (187 * 223-23 * 148) \text { mod } 256 \\ & 153(\mathrm{D} \neq 0 \text { and } \mathrm{D} \neq \text { Even })\end{array}$

Since determinants are not zero or even, we can use the key pair as keys for Hill Cipher.

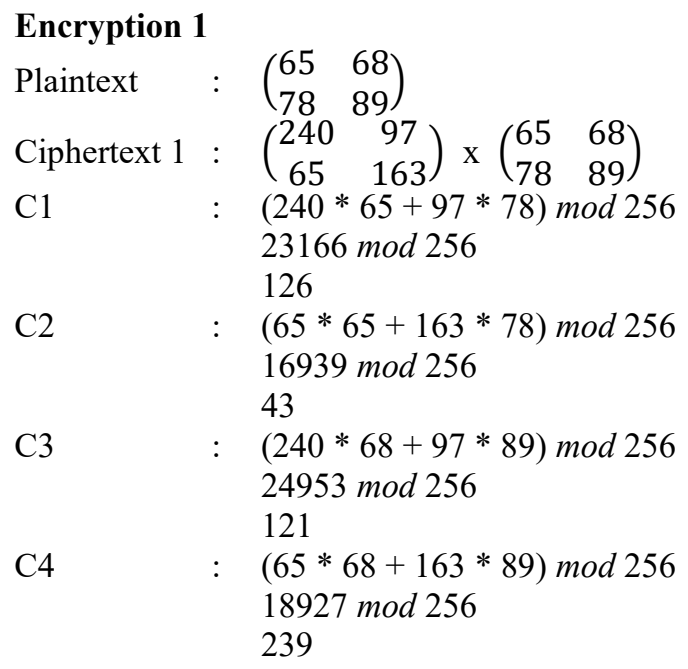

$\begin{array}{ll}\text { Ciphertext } 1: & \left(\begin{array}{cc}126 & 121 \\ 43 & 239\end{array}\right)^{T} \\ \text { Ciphertext } 1^{\mathrm{T}}: & \left(\begin{array}{cc}126 & 43 \\ 121 & 239\end{array}\right)\end{array}$

\section{Encryption 2}

$\begin{array}{ll}{\text { Ciphertext } 1^{\mathrm{T}}:}: & \left(\begin{array}{cc}126 & 43 \\ 121 & 239\end{array}\right) \\ \text { Ciphertext 2 : } & \left(\begin{array}{cc}187 & 23 \\ 148 & 223\end{array}\right) \times\left(\begin{array}{cc}126 & 43 \\ 121 & 239\end{array}\right) \\ \mathrm{C} 1 \quad & (187 * 126+23 * 121) \bmod 256 \\ & 26345 \bmod 256 \\ & 233 \\ \mathrm{C} 2 \quad & (148 * 126+223 * 121) \bmod 256 \\ & 45631 \bmod 256 \\ & 63 \\ \mathrm{C} 3 \quad & (187 * 43+23 * 239) \bmod 256 \\ & 13538 \bmod 256 \\ & 226 \\ \mathrm{C} 4 \quad & (148 * 43+223 * 239) \bmod 256 \\ & 59661 \bmod 256 \\ & 13 \\ & \left(\begin{array}{cc}233 & 226 \\ 63 & 13\end{array}\right) \\ \text { Ciphertext } 2: & \left(\begin{array}{ll}233 & 63 \\ 226 & 13\end{array}\right) \\ \text { Ciphertext } 2^{\mathrm{T}}:\end{array}$

Ciphertext $2^{\mathrm{T}}$ is the final result of the encryption the both methods. And for the decryption, we do the same way as easrlier. We see the explanation below:

\section{Decryption 1}

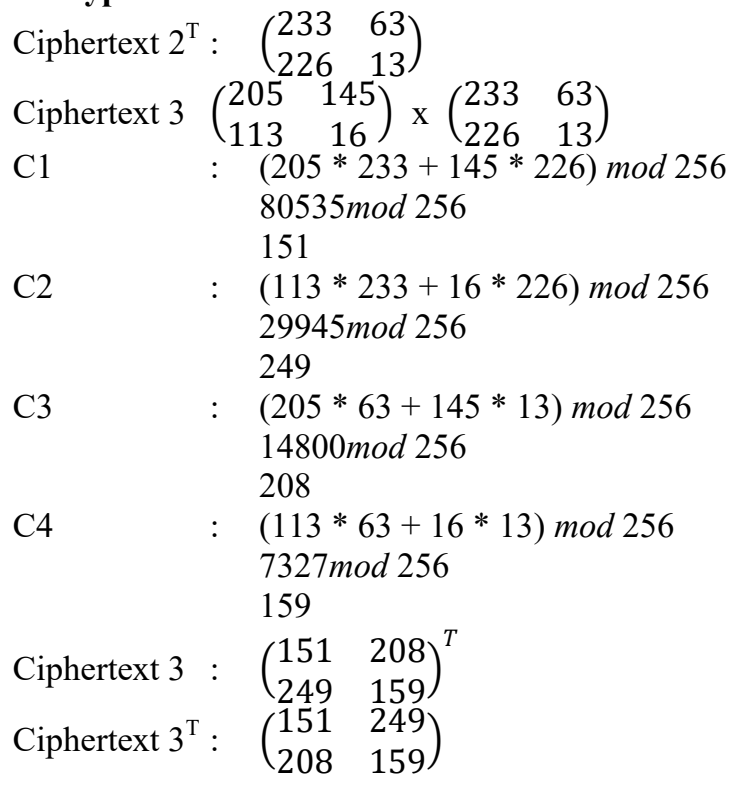

Decryption 2

$\begin{array}{lll}\text { Ciphertext } 3^{\mathrm{T}}: & \left(\begin{array}{cc}151 & 249 \\ 208 & 159\end{array}\right) \\ \text { Plaintext } \quad: & \left(\begin{array}{ll}55 & 209 \\ 76 & 115\end{array}\right) \times\left(\begin{array}{ll}151 & 249 \\ 208 & 159\end{array}\right) \\ \text { P1 } & : & (55 * 151+209 * 208) \bmod 256 \\ & 51777 \bmod 256 \\ & 65 \\ \text { P2 } \quad & (76 * 151+115 * 208) \bmod 256 \\ & 35396 \bmod 256 \\ \text { P3 } & 68 \\ & : & (55 * 249+209 * 159) \bmod 256 \\ & 46926 \bmod 256 \\ & 78\end{array}$




\section{International Journal of Science and Research (IJSR) \\ ISSN (Online): 2319-7064}

Index Copernicus Value (2013): 6.14 | Impact Factor (2015): 6.391

P4

$76 * 249+115 * 159) \bmod 256$

$37209 \bmod 256$

89

$\begin{array}{lll}\text { Plaintext } & :\left(\begin{array}{ll}65 & 78 \\ 68 & 89\end{array}\right)^{T} \\ \text { Plaintext }^{\mathrm{T}} & :\left(\begin{array}{ll}65 & 68 \\ 78 & 89\end{array}\right)\end{array}$

Plaintext ${ }^{\mathrm{T}}$ is the final result of the decryption the both methods.

After calculation, we can see the plaintext is turned into three parts of ciphertexts before finally turned back into plaintext again. Each participant needs to perform two stage of calculation where the sender does the encryption and decryption.For example in Table 1, it shows the complete work of encryption and decryption processes. The sentence is "THE QUICK BROWN FOX JUMPS OVER THE LAZY DOG". There are 40 characters. The PT1 shows the ASCII code of the characters, CT1, CT2 and CT3 is the three-pass protocol processes. The PT2 is the decryption of the ciphertext.

Table 1:Sample of Three-Pass Protocol in Hill Cipher

\begin{tabular}{|c|c|c|c|c|c|}
\hline NO. & PT1 & CT1 & CT2 & CT3 & PT2 \\
\hline 1 & 84 & 228 & 5 & 115 & 84 \\
\hline 2 & 72 & 179 & 225 & 97 & 72 \\
\hline 3 & 69 & 220 & 63 & 240 & 69 \\
\hline 4 & 32 & 135 & 123 & 248 & 32 \\
\hline 5 & 81 & 198 & 212 & 185 & 81 \\
\hline 6 & 85 & 24 & 131 & 252 & 85 \\
\hline 7 & 73 & 11 & 42 & 167 & 73 \\
\hline 8 & 67 & 179 & 20 & 194 & 67 \\
\hline 9 & 75 & 253 & 102 & 228 & 75 \\
\hline 10 & 32 & 228 & 140 & 67 & 32 \\
\hline 11 & 66 & 169 & 123 & 14 & 66 \\
\hline 12 & 82 & 246 & 81 & 242 & 82 \\
\hline 13 & 79 & 30 & 154 & 224 & 79 \\
\hline 14 & 87 & 34 & 32 & 91 & 87 \\
\hline 15 & 78 & 213 & 124 & 190 & 78 \\
\hline 16 & 32 & 186 & 153 & 133 & 32 \\
\hline 17 & 70 & 151 & 150 & 148 & 70 \\
\hline 18 & 79 & 72 & 28 & 234 & 79 \\
\hline 19 & 88 & 162 & 109 & 46 & 88 \\
\hline 20 & 32 & 72 & 110 & 109 & 32 \\
\hline 21 & 74 & 197 & 79 & 183 & 74 \\
\hline 22 & 85 & 251 & 141 & 11 & 85 \\
\hline 23 & 77 & 142 & 202 & 234 & 77 \\
\hline 24 & 80 & 95 & 169 & 111 & 80 \\
\hline 25 & 83 & 181 & 111 & 183 & 83 \\
\hline 26 & 32 & 187 & 141 & 8 & 32 \\
\hline 27 & 79 & 129 & 90 & 74 & 79 \\
\hline 28 & 86 & 149 & 88 & 118 & 86 \\
\hline 29 & 69 & 9 & 70 & 250 & 69 \\
\hline 30 & 82 & 92 & 222 & 15 & 82 \\
\hline 31 & 32 & 7 & 151 & 48 & 32 \\
\hline 32 & 84 & 96 & 213 & 10 & 84 \\
\hline 33 & 72 & 39 & 122 & 240 & 72 \\
\hline 34 & 69 & 68 & 208 & 248 & 69 \\
\hline 35 & 32 & 216 & 153 & 142 & 32 \\
\hline 36 & 76 & 96 & 232 & 187 & 76 \\
\hline 37 & 65 & 69 & 139 & 137 & 65 \\
\hline 38 & 90 & 255 & 179 & 220 & 90 \\
\hline 39 & 89 & 155 & 206 & 180 & 89 \\
\hline 40 & 32 & 163 & 180 & 110 & 32 \\
\hline & & & & \\
\hline
\end{tabular}

The use of Three-Pass Protocol on Hill Cipher is very useful way to improve the data security level in the process of sending a message. Besides improving the security, this method also stops distributing keys between sender and receiver. If someone wants to take the information, it will be suspended.In Table 1, we see there are three ciphertext produced. Someone might be intercepting the information. But actually, he does not have the keys since they are not transferred. It is hard to break the hidden information since the key is not provided. But in the conventional method, the key is distributed as well. It really makes the key vulnerable.

\section{Conclusion}

We concludethat Three-Pass Protocol can be applied in Hill Cipher encryption. It helps the sender to give more protection to their data from being intercepted. The undistributed key system is more secure since the both participants do not have to exchange key when doing this process. Three-Pass Protocol is the best technique to gain the information security more.

\section{References}

[1] A. A. Abdullah, R. Khalaf dan M. Riza, "A Realizable Quantum Three-Pass Protocol Authentication," Mathematical Problems in Engineering, 2015.

[2] R. Kumar dan R. C., "Analysis of Diffie Hellman Key Exchange Algorithm with Proposed Key Exchange Algorithm," International Journal of Emerging Trends \& Technology in Computer Science, vol. 4, no. 1, pp. 4043, 2015.

[3] M. Ahmed, B. Sanja, D. Aldiaz, A. Rezaei dan H. Omotunde, "Diffie-Hellman and Its Application in Security Protocols," International Journal of Engineering Science and Innovative Technology, vol. 1, no. 2, pp. 6973, 2008.

[4] C. Stubbs, "Three-Pass Protocol," 20 November 2013. [Online]. Available: http://asymmetriccryptography.blogspot.co.id/. [Diakses 1 May 2016].

[5] M. N. A. Rahman, A. F. A. Abidin, M. K. Yusof dan N. S. M. Usop, "Cryptography: A New Approach of Classical Hill Cipher," International Journal of Security and Its Applications, vol. 7, no. 2, pp. 179-190, 2013.

[6] S. I. Chowdhury, S. A. M. Shohag dan H. Sahid, "A Secured Message Transaction Approach by Dynamic Hill Cipher Generation and Digest Concatenation," International Journal of Computer Applications, vol. 23, no. 9, pp. 25-31, 2011.

[7] A. A. Khalaf, M. S. A. El-karim dan H. F. A. Hamed, “A Triple Hill Cipher Algorithm Proposed to Increase the Security of Encrypted Binary Data and its Implementation Using FPGA," ICACT Transactions on Advanced Communications Technology, vol. 5, no. 1, pp. 752-757, 2016.

[8] J. Chase dan M. Davis, "Extending the Hill Cipher," 2010.

[9] A. P. U. Siahaan, "RC4 Technique in Visual Cryptography RGB Image Encryption," International Journal of Computer Science and Engineering, vol. 3, no. 7, pp. 1-6, 2016 


\section{International Journal of Science and Research (IJSR) \\ ISSN (Online): 2319-7064}

Index Copernicus Value (2013): 6.14 | Impact Factor (2015): 6.391

\section{Author Profile}

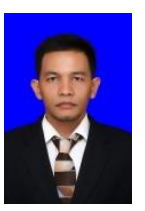

Andysah Putera Utama Siahaan was born in Medan, Indonesia, in 1980. He received the S.Kom. degree in computer science from Universitas Pembangunan Panca Budi, Medan, Indonesia, in 2010, and the M.Kom. in computer science as well from the University of Sumatera Utara, Medan, Indonesia, in 2012. In 2010, he joined the Department of Engineering, Universitas Pembangunan Panca Budi, as a Lecturer, and in 2012 became a junior researcher. He is applying for his $\mathrm{Ph}$. D. degree in 2016. He has written in several international journal and conference. He is now active in writing papers and joining conferences. 